\title{
Comparison of postoperative paresthesia after sagittal split osteotomy among different fixation methods: a one year follow-up study
}

\author{
Reza Tabrizi ${ }^{1,2}$, Kousha Bakrani ${ }^{2}$, Farshid Bastami ${ }^{1,3}$ \\ ${ }^{1}$ Oral and Maxillofacial Surgery Department, School of Dentistry, Shahid Beheshti University of Medical Sciences, Tehran, \\ ${ }^{2}$ Oral and Maxillofacial Surgery Department, School of Dentistry, Shiraz University of Medical Sciences, Shiraz, \\ ${ }^{3}$ Dental Research Center, Research Institute of Dental Sciences, School of Dentistry, Shahid Beheshti University of Medical Sciences, Tehran, Iran
}

\begin{abstract}
J Korean Assoc Oral Maxillofac Surg 2019;45:215-219)
Objectives: Postoperative paresthesia is a common complication after sagittal split osteotomy (SSO). This study aimed to compare paresthesia among different fixation methods one year postoperative.

Materials and Methods: This prospective cohort study assessed subjects in four groups: class II with miniplate fixation (Group 1), class II with three-screw fixation (Group 2), class III with miniplate fixation (Group 3), and class III with three-screw fixation (Group 4). Paresthesia was evaluated one year postoperative based on a 0-10 visual analogue scale. Pearson correlation was used to evaluate associations of age and mandibular movement with paresthesia. ANOVA was used to compare paresthesia among groups.

Results: A total of 80 subjects were enrolled, with 20 subjects in each of the four groups. The Pearson correlation test demonstrated a significant correlation between mandibular movement and paresthesia $(P=0.001)$. Comparison of paresthesia among the groups showed significant differences among groups 1 and 2, 2 and 3 , and 3 and $4(P<0.05)$.

Conclusion: The three-screw fixation method led to more paresthesia one year postoperative compared with miniplate fixation. In addition, the magnitude of mandibular movement had a positive correlation with paresthesia.
\end{abstract}

Key words: Mandible, Paresthesia, Sagittal split osteotomy, Skeletal deformity, Inferior alveolar nerve

[paper submitted 2019. 1. 15 / revised 2019. 5. 8 / accepted 2019. 5. 12]

\section{Introduction}

Sagittal split osteotomy (SSO) is the most commonly performed osteotomy for correction of class II or class III skeletal deformities. One of the common complications of SSO is inferior alveolar nerve (IAN) injury, which presents as postoperative numbness in the lower lip and mental area ${ }^{1}$. The prevalence of postoperative paresthesia following SSO ranges from $9 \%$ to $84.6 \%^{2}$. IAN injury defined as both direct

\footnotetext{
Farshid Bastami

Dental Research Center, Research Institute of Dental Sciences, and Oral and Maxillofacial Surgery Department, School of Dentistry, Shahid Beheshti University of Medical Sciences, Velenjak St., Shahid Chamran Highway, Tehran 1983963113, Iran

TEL: +98-9126704434 FAX: +98-2122427753

E-mail: farshidbastami@sbmu.ac.ir

ORCID: https://orcid.org/0000-0002-6332-4032

(c) This is an open-access article distributed under the terms of the Creative Commons Attribution Non-Commercial License (http://creativecommons.org/ licenses/by-nc/4.0/), which permits unrestricted non-commercial use, distribution, and reproduction in any medium, provided the original work is properly cited.

Copyright (C) 2019 The Korean Association of Oral and Maxillofacial Surgeons. All rights reserved.
}

and indirect operative trauma. This trauma results in changes in sensibility or other forms of altered sensation of the lower lip and/or mental region ${ }^{3}$. In addition, injury to the IAN is unpredictable even when surgery is performed by an expert surgeon ${ }^{4}$.

Sensory disturbances following SSO have the potential to affect patient performance because of their effects on speech, eating, drinking, and social interactions ${ }^{5}$. Patient complaints regarding sensory disturbances are important issues faced by surgeons after SSO. Reported paresthesia after SSO can be considered a determining factor of the severity of nerve injury after osteotomy ${ }^{6}$.

Various factors have been suggested to be responsible for the development of IAN injury after SSO, including the fixation method, patient age, the surgical procedure, improper splinting, magnitude of mandibular movement, experience of the surgeon, and timing of postoperative neurosensory evaluation ${ }^{4}$. In addition, the IAN may be distracted or compressed based on movement of the distal segment of the mandible in class II or class III deformities. The fixation method (po- 
sitional screws or miniplates) may affect the frequency of paresthesia $^{7}$. The placement of semi-rigid fixation plates and screws may result in nerve injury either directly or through compression of the nerve between bony segments after fixation $^{8}$.

Clinical diagnosis of neurosensory disturbances after SSO is mostly based on clinical sensory testing. Examinations can be either mechanoceptive or nociceptive ${ }^{9}$. Clinical examination is sometimes difficult because of the heterogeneity of the methods, variability in the definitions of neurosensory disturbance, and change over time during follow-up ${ }^{10}$.

The reliability of subjective reporting is controversial. Subjective reports are used widely to evaluate postoperative paresthesia because of the lack of objective factors in the diagnosis of sensory nerve damage ${ }^{11}$. It has been suggested that paresthesia and sensory disturbance after SSO are primarily overestimated, but may be underestimated with increasing time from surgery. It seems that patients adapt or become accustomed to what they consider "normal" over time. Normal sensation was different among patients in various studies ${ }^{12}$. Debate continues over which type of evaluation (sensory testing or patient reporting) should be used to assess nerve injury and recovery ${ }^{13}$. If evaluation is used for decision-making regarding microsurgical nerve repair, electrophysiological assessment of nerve integrity or stimulus detection measures may be valuable. Patient adaptation to altered sensitivity ${ }^{14,15}$ or the effects of medication ${ }^{16}$ can be evaluated by stimulus perception or self-report ${ }^{13}$.

We hypothesized that paresthesia occurs in patients with class II skeletal deformities more than in patients with class III deformities and also in patients in whom positional screws are used for fixation. The aim of this study was to compare the amount of postoperative paresthesia in patients with class II and class III skeletal deformities with different fixation methods including the use of positional screws or miniplates.

\section{Materials and Methods}

The authors designed a prospective cohort study. The sample was derived from a population of patients treated at the Oral and Maxillofacial Surgery Department of Shiraz University of Medical Sciences (Shiraz, Iran) between September 1, 2012 and October 31, 2014. The research was approved by the Medical Ethics Committee of Shiraz University of Medical Sciences (approval No. 8793062). Also, all procedures followed the policies and principles of the Declaration of Helsinki during all clinical procedures.

Subjects eligible for inclusion in the study had skeletal class II or class III deformities and underwent SSO. Subjects were excluded from the study if they had asymmetry, previous trauma or fracture of the mandible, previous orthognathic surgery, surgical augmentation or concomitant genioplasty, or took any psychological or anti-inflammatory drugs. None of the subjects had impacted mandibular third molars or had undergone third molar surgery during the past year. Subjects who had bad splits during osteotomy were excluded from the study.

Subjects were divided into four groups; Group 1: patients with a class II skeletal deformity that underwent miniplate fixation of each osteotomy site, Group 2: patients with a class II skeletal deformity that underwent fixation by three positional screws at each osteotomy site, Group 3: patients with a class III skeletal deformity that underwent miniplate fixation at each osteotomy site, and Group 4: patients with a class III skeletal deformity that underwent fixation by three positional screws at each osteotomy site.

The amount of mandibular movement was assessed via lateral cephalogram. The change in the distance between the upper and lower incisors was measured before and immediately after surgery.

All patients were followed one year postoperative.(Table 1) To assess the level of paresthesia, visual analogue scale scores were interpreted as follows: 0 to 2 mild discomfort, 2 to 4 mild to moderate discomfort, 4 to 6 moderate discom-

Table 1. Sex and age of the patients and the amount of mandibular movement in the four groups

\begin{tabular}{lccccc}
\hline \multicolumn{1}{c}{ Variable } & Group 1 & Group 2 & Group 3 & Group 4 & $P$-value \\
\hline Sex (M/F) & $6 / 14$ & $7 / 13$ & $7 / 13$ & $9 / 11$ & 0.35 \\
Age (yr) & $22.85 \pm 3.51$ & $23.10 \pm 5.05$ & $22.80 \pm 3.90$ & $22.25 \pm 4.49$ & 0.93 \\
Mandibular movement (mm) & $4.45 \pm 0.94$ & $4.00 \pm 0.86$ & $4.10 \pm 0.85$ & $3.95 \pm 0.86$ & 0.28 \\
\hline
\end{tabular}

(M: male, F: female)

Values are presented as number only or mean \pm standard deviation.

Reza Tabrizi et al: Comparison of postoperative paresthesia after sagittal split osteotomy among different fixation methods: a one year follow-up study. J Korean Assoc Oral Maxillofac Surg 2019 
fort, 6 to 8 moderate to severe discomfort, and 8 to 10 severe to intolerable discomfort ${ }^{2}$.

\section{Surgical approach}

The surgical method described by Epker ${ }^{17}$ was followed. An incision was made over the anterior portion of the vertical ramus, extending to the mesial aspect of the first molar.

Subperiosteal dissection was carried down to the inferior border of the mandible, where a lateral channel retractor was placed. A long bur was used to make a horizontal bone cut through the medial cortex of the ramus, just above and approximately posterior to the lingula. The medial osteotomy line was extended 3 to $4 \mathrm{~mm}$ beyond the lingula. The vertical cut was made through the buccal cortex, distal to the second molar or further anterior. The two osteotomies were then connected with a \#701 fissure bur. A spreader and a narrow osteotome were used to gently lift the lateral cortex, and the osteotome was used to create a step along the connecting cut to ensure that the split remained close to the lateral cortex. Patients in groups 1 and 3 underwent fixation with a fourhole miniplate with a bar and four screws at each osteotomy site. In groups 2 and 4, patients underwent fixation with three positional screws using a trocar.

\section{Statistical analysis}

Statistical analyses were performed using the IBM SPSS Statistics software (ver. 19; IBM Co., Armonk, NY, USA). ANOVA was used to compare age, the amount of mandibular movement, and the level of paresthesia among groups. The chi-square test was applied to compare sex among groups. The Pearson correlation test was used to assess associations

Table 2. Pairwise comparison of the four groups by post hoc test

\begin{tabular}{ccc}
\hline \multicolumn{2}{c}{ Groups } & $P$-value \\
\hline Group 4 & 1 & 0.16 \\
& 2 & 0.67 \\
Group 1 & 3 & 0.04 \\
& 2 & 0.01 \\
Group 2 & 3 & 0.92 \\
& 4 & 0.16 \\
Group 3 & 1 & 0.01 \\
& 3 & 0.001 \\
& 4 & 0.67 \\
& 1 & 0.92 \\
& 4 & 0.001 \\
\hline
\end{tabular}

Reza Tabrizi et al: Comparison of postoperative paresthesia after sagittal split osteotomy among different fixation methods: a one year follow-up study. J Korean Assoc Oral Maxillofac Surg 2019 of age and amount of mandibular movement with paresthesia. Pairwise comparison of groups in terms of paresthesia was performed using Scheffe's post hoc test. We considered $P$-values $<0.05$ statistically significant.

\section{Results}

Eighty subjects were divided into four groups with 20 subjects in each group. Mean age ( \pm standard deviation) was $22.76 \pm 4.21$ years and mean mandibular movement was $4.12 \pm 0.89 \mathrm{~mm}$. Analysis of the data did not demonstrate a significant difference in sex, age, or mandibular movement among the studied groups $(P<0.05)$. (Table 1$)$ The mean level of paresthesia was $3.12 \pm 2.15$. Pearson correlation demonstrated a significant correlation between mandibular movement and paresthesia $(P=0.001)$. There was no correlation between age and paresthesia $(P>0.05)$.

The mean level of paresthesia was $2.82 \pm 1.87$ in males and $3.30 \pm 2.30$ in females $(P>0.05)$. The mean level of paresthesia was 2.40 \pm 1.70 in Group 1, 4.40 \pm 2.23 in Group 2, $2.00 \pm 1.29$ in Group 3, and 3.70 \pm 2.40 in Group 4. Comparison of paresthesia among these groups showed a significant difference $(P<0.05)$. Further post hoc testing revealed a significant difference between groups 1 and 2, groups 2 and 3, and groups 3 and 4.(Table 2)

\section{Discussion}

Nerve manipulation and retraction for the medial osteotomy cut, the actual splitting procedure, excessive stretching of the nerve during segment manipulation, and compression of the nerve during fixation have been considered factors responsible for nerve disturbance during $\mathrm{SSO}^{13}$. In the current study, we evaluated paresthesia among different skeletal deformities and fixation methods in four groups one year postoperative. The groups had similar age and sex characteristics, and similar mandibular movement.

In the current study, mean paresthesia was higher in screwtype fixations than plate fixations used for both setback and advancement of the mandible. Screw-type fixations are used bicortically, while plates are typically fixed monocortically. An animal study on monkeys showed that nerve injury occurred in both monocortical and bicortical fixation during setback of the mandible via SSO. Although considerable recovery was detected after both forms of fixation at 12 weeks, nerve function was reported to be much better after plate fixation than after screw fixation ${ }^{18}$. 
In the current study, we found a positive correlation between the amount of mandibular movement and postoperative paresthesia. Anatomical characteristics of the mandible such as the intrabony course of the mandibular canal or thickness of the bone at the osteotomy site may also increase the risk of nerve disturbance during $\mathrm{SSO}^{19}$. The close contact between the mandibular canal and the external cortical bone increases the risk of neurosensory disturbances as well ${ }^{20}$. The prevalence of nerve injury after SSO in class III cases is dependent on the position of the mandibular canal and the size of the mandibular angle ${ }^{21}$. Mandibular anatomy varies in retrognathia and prognathia. Individuals with retrognathia have a small mandible with a short body and the IAN is stretched during SSO for mandibular advancement. In subjects with prognathia, the mandible is huge with a long body and the IAN is not stretched but may be twisted during SSO. These characteristics may lead to differences in the frequency of nerve disturbance between these two types of deformities. In our study, no differences were detected between class II and class III skeletal patients with regard to postoperative paresthesia, but a positive correlation was seen between the amount of mandibular movement and paresthesia. Ylikontiola et al. ${ }^{22}$ considered age, the intraoperative magnitude of mandibular movement, and the degree of manipulation of the IAN important factors in nerve dysfunction after SSO.

Clinical neurosensory testing generally consists of two basic types according to the specific receptors stimulated through nociceptive, mechanoceptive, and cutaneous contact. Mechanoceptive testing consists of the two-point discrimination test (TPD), static light touch, and brush directional stroke tests. The pin-prick and thermal discrimination tests are a subgroup of the nociceptive testing group ${ }^{23}$. In this study, paresthesia was evaluated subjectively (self-reported). The absence of objective data is a main limitation of this study. Improvement of paresthesia in patients who underwent bilateral SSO (BSSO) was attributed to recovery of the IAN or adaptation to changes in nerve function, especially in bilateral paresthesia. It is a disadvantage of self-reported paresthesia as an evaluation test in neurosensory paresthesia. Therefore, future studies are needed to investigate paresthesia objectively.

\section{Conclusion}

According to the results of this study, bicortical fixation by screws can be considered a risk factor for nerve disturbance. Furthermore, although the type of skeletal deformity had no effect on postoperative paresthesia, the amount of mandibular movement had a positive correlation with paresthesia.

\section{ORCID}

Reza Tabrizi, https://orcid.org/0000-0001-7204-7746

Kousha Bakrani, https://orcid.org/0000-0002-3178-8174

Farshid Bastami, https://orcid.org/0000-0002-6332-4032

\section{Authors' Contributions}

R.T., K.B., and F.B. participated in data collection and wrote the manuscript. R.T., K.B., and F.B. participated in the study design and performed the statistical analysis. R.T. participated in the study design and coordination and helped to draft the manuscript. All authors read and approved the final manuscript.

\section{Acknowledgements}

The present study was supported by a grant (No. 8793062) from Shiraz University of Medical Sciences. We would like thank the vice chancellery of the Shiraz University of Medical Science for supporting this research.

\section{Ethics Approval and Consent to Participate}

The research was approved by the Medical Ethics Committee of Shiraz University of Medical Sciences (approval No. 8793062).

\section{Conflict of Interest}

No potential conflict of interest relevant to this article was reported.

\section{References}

1. Kim SG, Park SS. Incidence of complications and problems related to orthognathic surgery. J Oral Maxillofac Surg 2007;65:2438-44.

2. Al-Bishri A, Barghash Z, Rosenquist J, Sunzel B. Neurosensory disturbance after sagittal split and intraoral vertical ramus osteotomy: as reported in questionnaires and patients' records. Int J Oral Maxillofac Surg 2005;34:247-51.

3. Bruckmoser E, Bulla M, Alacamlioglu Y, Steiner I, Watzke IM. Factors influencing neurosensory disturbance after bilateral sagittal split osteotomy: retrospective analysis after 6 and 12 months. Oral Surg Oral Med Oral Pathol Oral Radiol 2013;115:473-82.

4. Kuroyanagi N, Miyachi H, Ochiai S, Kamiya N, Kanazawa T, Nagao T, et al. Prediction of neurosensory alterations after sagittal split ramus osteotomy. Int J Oral Maxillofac Surg 2013;42:814-22. 
5. Strauss ER, Ziccardi VB, Janal MN. Outcome assessment of inferior alveolar nerve microsurgery: a retrospective review. J Oral Maxillofac Surg 2006;64:1767-70.

6. Politis C, Sun Y, Lambrichts I, Agbaje JO. Self-reported hypoesthesia of the lower lip after sagittal split osteotomy. Int J Oral Maxillofac Surg 2013;42:823-9.

7. Westermark A, Bystedt $\mathrm{H}$, von Konow L. Inferior alveolar nerve function after mandibular osteotomies. Br J Oral Maxillofac Surg 1998;36:425-8.

8. Phillips C, Essick G. Inferior alveolar nerve injury following orthognathic surgery: a review of assessment issues. J Oral Rehabil 2011;38:547-54.

9. Antonarakis GS, Christou P. Quantitative evaluation of neurosensory disturbance after bilateral sagittal split osteotomy using Semmes-Weinstein monofilaments: a systematic review. J Oral Maxillofac Surg 2012;70:2752-60.

10. Poort LJ, van Neck JW, van der Wal KG. Sensory testing of inferior alveolar nerve injuries: a review of methods used in prospective studies. J Oral Maxillofac Surg 2009;67:292-300.

11. Teerijoki-Oksa T, Jääskeläinen S, Forssell K, Virtanen A, Forssell $\mathrm{H}$. An evaluation of clinical and electrophysiologic tests in nerve injury diagnosis after mandibular sagittal split osteotomy. Int J Oral Maxillofac Surg 2003;32:15-23.

12. Jacks SC, Zuniga JR, Turvey TA, Schalit C. A retrospective analysis of lingual nerve sensory changes after mandibular bilateral sagittal split osteotomy. J Oral Maxillofac Surg 1998;56:700-4; discussion 705

13. Roychoudhury S, Nagori SA, Roychoudhury A. Neurosensory disturbance after bilateral sagittal split osteotomy: a retrospective study. J Oral Biol Craniofac Res 2015;5:65-8.

14. Essick GK, Phillips C, Kim SH, Zuniga J. Sensory retraining following orthognathic surgery: effect on threshold measures of sensory function. J Oral Rehabil 2009;36:415-26.

15. Phillips C, Kim SH, Essick G, Tucker M, Turvey TA. Sensory retraining after orthognathic surgery: effect on patient report of altered sensations. Am J Orthod Dentofacial Orthop 2009;136:78894.

16. Seo K, Tanaka Y, Terumitsu M, Someya G. Efficacy of steroid treatment for sensory impairment after orthognathic surgery. J Oral Maxillofac Surg 2004;62:1193-7.

17. Epker BN. Modifications in the sagittal osteotomy of the mandible. J Oral Surg 1977;35:157-9.

18. Hu J, Zhao Q, Tang J, Zheng Z, Qi MC. Changes in the inferior alveolar nerve following sagittal split ramus osteotomy in monkeys: a comparison of monocortical and bicortical fixation. Br J Oral Maxillofac Surg 2007;45:265-71.

19. Rajchel J, Ellis E 3rd, Fonseca RJ. The anatomical location of the mandibular canal: its relationship to the sagittal ramus osteotomy. Int J Adult Orthodon Orthognath Surg 1986;1:37-47.

20. Yamamoto R, Nakamura A, Ohno K, Michi KI. Relationship of the mandibular canal to the lateral cortex of the mandibular ramus as a factor in the development of neurosensory disturbance after bilateral sagittal split osteotomy. J Oral Maxillofac Surg 2002;60:490-5.

21. Yamauchi K, Takahashi T, Kaneuji T, Nogami S, Yamamoto N, Miyamoto I, et al. Risk factors for neurosensory disturbance after bilateral sagittal split osteotomy based on position of mandibular canal and morphology of mandibular angle. J Oral Maxillofac Surg 2012;70:401-6.

22. Ylikontiola L, Kinnunen J, Oikarinen K. Factors affecting neurosensory disturbance after mandibular bilateral sagittal split osteotomy. J Oral Maxillofac Surg 2000;58:1234-9; discussion 123940.

23. Akal ÜK, Sayan NB, Aydoğan S, Yaman Z. Evaluation of the neurosensory deficiencies of oral and maxillofacial region following surgery. Int J Oral Maxillofac Surg 2000;29:331-6.

How to cite this article: Tabrizi R, Bakrani K, Bastami F. Comparison of postoperative paresthesia after sagittal split osteotomy among different fixation methods: a one year follow-up study. J Korean Assoc Oral Maxillofac Surg 2019;45:215-219. https://doi. org/10.5125/jkaoms.2019.45.4.215 